\title{
Measurement of Peripheral Plasma Turbulence Using a Fast Camera in Heliotron $\mathbf{J}$
}

\author{
Nobuhiro NISHINO, Tohru MIZUUCHI ${ }^{1)}$, Shinichi KOBAYASHI ${ }^{1)}$, Kazunobu NAGASAKI ${ }^{1)}$, \\ Hiroyuki OKADA $^{1)}$, Fumimichi SANO ${ }^{1)}$, Satoru YAMAMOTO ${ }^{2)}$ and Katsumi KONDO ${ }^{3)}$ \\ Graduate School of Engineering, Hiroshima University, Kagamiyama, Higashi-Hiroshima 739-8527, Japan \\ ${ }^{1)}$ Institute of Advanced Energy, Kyoto University, Gokasho, Uji 611-0011, Japan \\ ${ }^{2)}$ Kyoto University Pioneering Research Unit for Next Generation, Yoshida, Sakyo-ku 606-8501, Kyoto, Japan \\ ${ }^{3)}$ Graduate School of Energy Science, Kyoto University, Gokasho, Uji 606-0011, Japan
}

(Received 16 November 2007 / Accepted 3 March 2008)

\begin{abstract}
Using a fast camera with tangential view, the motion of the filamentary structures in peripheral regions was observed. In the L-mode, the filamentary structures are relatively thinner than those of the H-mode. The direction of filamentary structure motion in the H-mode plasma was changed opposite to that in the L-mode plasma, and the motion speed was also doubled. During L-H transition, this motion was stopped. If this motion is poloidal rotation due to $E_{\mathrm{r}} \times B$ drift, $E_{\mathrm{r}}$ should be positive in the L-mode plasma and negative in the H-mode plasma.
\end{abstract}

(c) 2008 The Japan Society of Plasma Science and Nuclear Fusion Research

Keywords: turbulence, peripheral plasma, L-H transition, H-mode, Heliotron J, fast camera

DOI: $10.1585 /$ pfr.3.S1023

\section{Introduction}

Study of peripheral turbulence is a very important issue for fusion research due to its relationship with the energy confinement and H-mode physics. Fast cameras have been installed in Heliotron $\mathrm{J}$ [1] to obtain information on the peripheral plasma turbulence since several years. In our previous work, using a fast camera (Ultima-SE, Photron) in combination with a small movable carbon target, the structure of a low-frequency $(5-6 \mathrm{kHz})$ edge plasma oscillation in high-electron density ECH discharges was observed just after L-H transition [2]. Also, a combination of a fast camera (FX-K4, NAC image technology), movable probe, and directional gas puff provided us with important information the spatial profiles of a turbulent burst in the edge plasma were observed as a filamentary structure in the Lmode [3-5]. Each burst of the ion saturation current corresponded to the filamentary structure that hit the probe simultaneously.

Recently, the turbulent structures during the L- and $\mathrm{H}$ modes were observed by the latter camera with/without directional gas puff [6]. It was clear that the spatial structure of peripheral turbulence during the L-mode was different from that of the H-mode. A relatively wide structure along the magnetic field was observed in the H-mode; however, a narrower structure along the magnetic field was observed in the L-mode. Moreover, these filamentary structures sometimes disappeared in the H-mode.

In this paper, the latest results of Heliotron J plasma easurement by the fast camera are reported.

\section{Experimental Setup}

The tangential port was provided with the fast camera to measure peripheral plasma behavior easily. Figure 1 shows the tangential port position (present experiment, green) and horizontal port position (past experiments, blue) for the fast camera.

Figure 2 shows a typical image of the fast camera from this tangential port. The image is the initial discharge phase of a Heliotron J plasma. The bright string in the image became thicker and darker, and the equilibrium state of Heliotron $\mathbf{J}$ plasma was obtained within a few $\mathrm{ms}$ after this image. The two top ports of Heliotron J vacuum vessel are seen clearly.

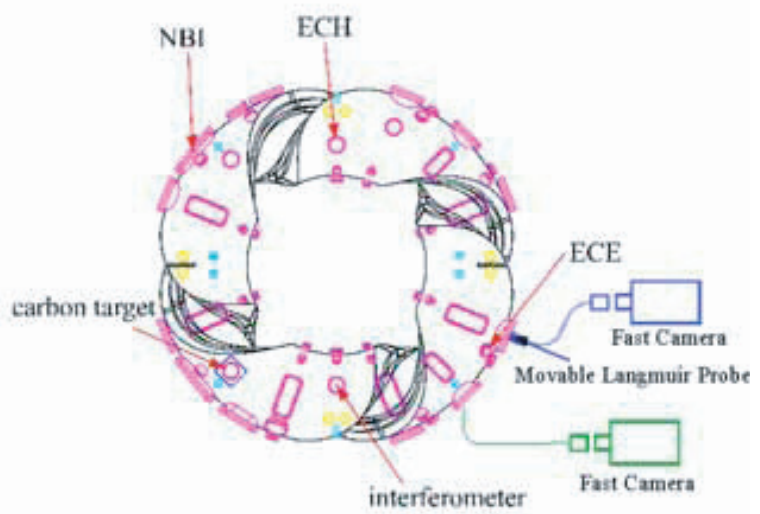

Fig. 1 Location of tangential view for the fast camera in Heliotron $\mathrm{J}$ 


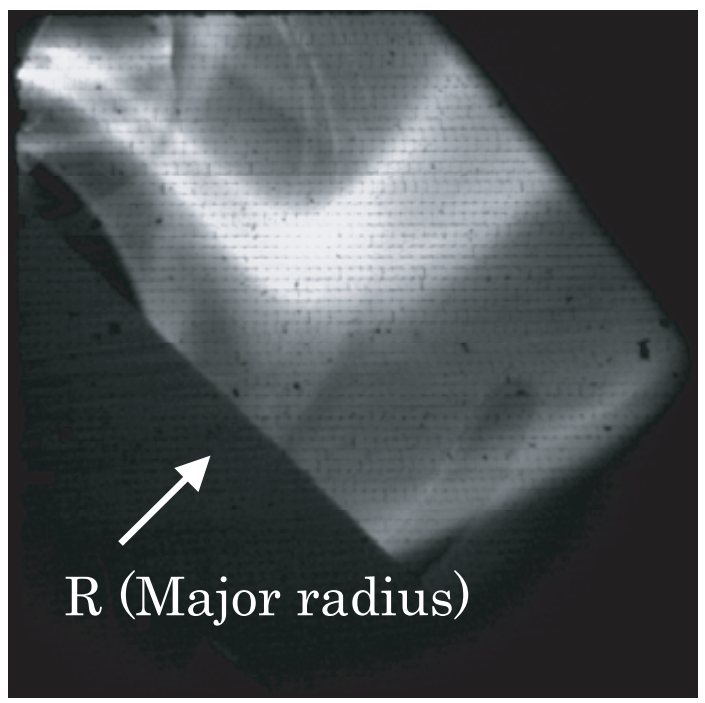

Fig. 2 Tangential view from the fast camera A part of the original image $(448 \times 368$ pixels, 10000 FPS $)$.

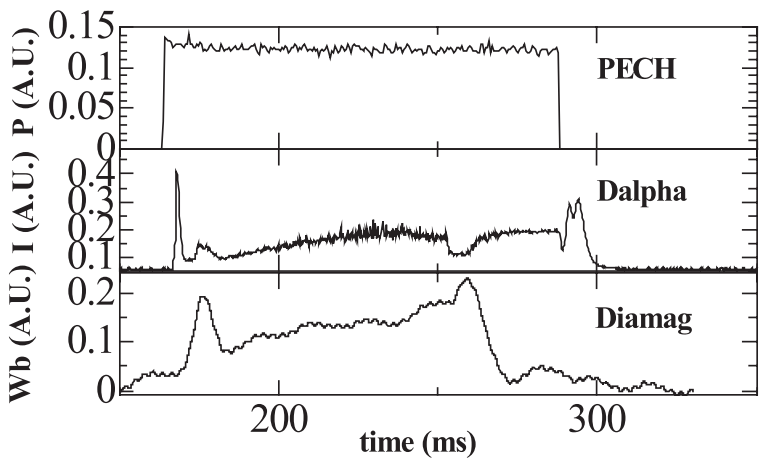

Fig. 3 Typical waveform of $\mathrm{H}$-mode in $\mathrm{ECH}$ discharge Upper:ECH power, Middle: D $\alpha$, Lower: diamagnetic signal.

\section{Results and Discussion}

In high-density ECH discharges, abrupt drops in the $\mathrm{D} \alpha$ signal were observed and H-mode plasmas were obtained. In these shots, the reproducibility was very good. A typical waveform of the H-mode shot is shown in Fig. 3.

The H-mode began at $252.8 \mathrm{~ms}$ and the diamagnetic signal increased rapidly after the L-H transition. Typical periods of the H-mode in these shots are about $5 \mathrm{~ms}$. During the $\mathrm{H}$-mode, the electron density also increased rapidly, and this increment is not controllable. Therefore, the hot plasma was extinguished with the radiation collapse after $\mathrm{H}$-mode in several shots.

Figures 4 (a) and (b) show the tangential camera images of the L-mode plasma and the H-mode plasma, respectively. The emission from the peripheral plasma region was relatively clear in the $\mathrm{H}$-mode (inside the white dashed ellipses), and the volume of the H-mode plasma was somewhat larger than that of the L-mode in the images. From this tangential view, the filamentary structures in the peripheral region were observed clearly in both

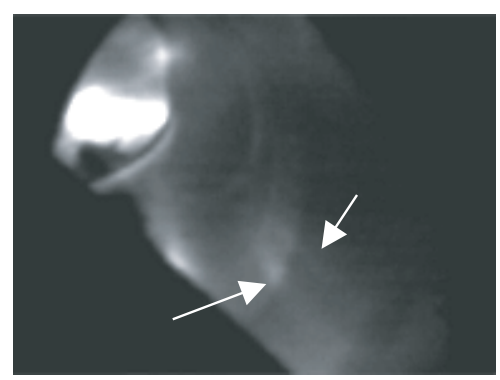

(a)

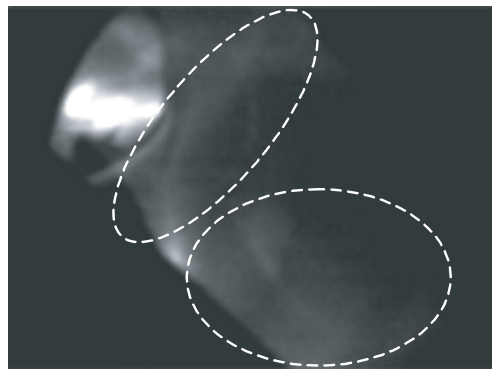

(b)

Fig. 4 Tangential views of the L-mode and H-mode plasmas (a) L-mode, (b) H-mode.

modes (two white arrows).

These filamentary structures are widely seen in various machines such as tokamaks/ST and stellarators, and it is believed that they are parallel to the magnetic field. In these H-mode experiments, the motion of the filamentary structures perpendicular to the magnetic field was recognized in both modes, and the motion during the L-mode was opposite to that of the H-mode. Moreover, the motion was stopped during the $\mathrm{L}-\mathrm{H}$ transition. If these motions were poloidal rotation, the rotation direction was counterclockwise during the L-mode and clockwise during the $\mathrm{H}$ mode.

The magnetic field direction and the camera viewing direction were counterclockwise at the horizontal plane (see Fig. 1) in these shots. Therefore, if the filamentary structures would rotate by $E_{\mathrm{r}} \times B$ drift, $E_{\mathrm{r}}$ should be positive in the L-mode and negative in the $\mathrm{H}$-mode in Heliotron $\mathrm{J}$ plasmas. These results were consistent with the past results on the H-mode review in Heliotron J [7]. To observe these motions easily, time-dependent FFT analysis is often used. The low-frequency $8.75 \mathrm{kHz}$ signal was relatively strong in the power spectra of the camera pixel data during the L-mode, and no strong peak was obtained in the power spectra during the H-mode (not shown in the figure). To evaluate the two-dimensional rotation effect, this low fluctuation frequency was chosen to observe how the filamentary structures in Heliotron $\mathbf{J}$ plasmas behave during the L- and H-modes. Figure 5 shows two-dimensional phase images using time-dependent FFT analysis from the original camera images. The frequency of the fluctuation signal depicted on each frame was $8.75 \mathrm{kHz}$, and the fast camera was operated at 40000 frames per second (FPS). We used 

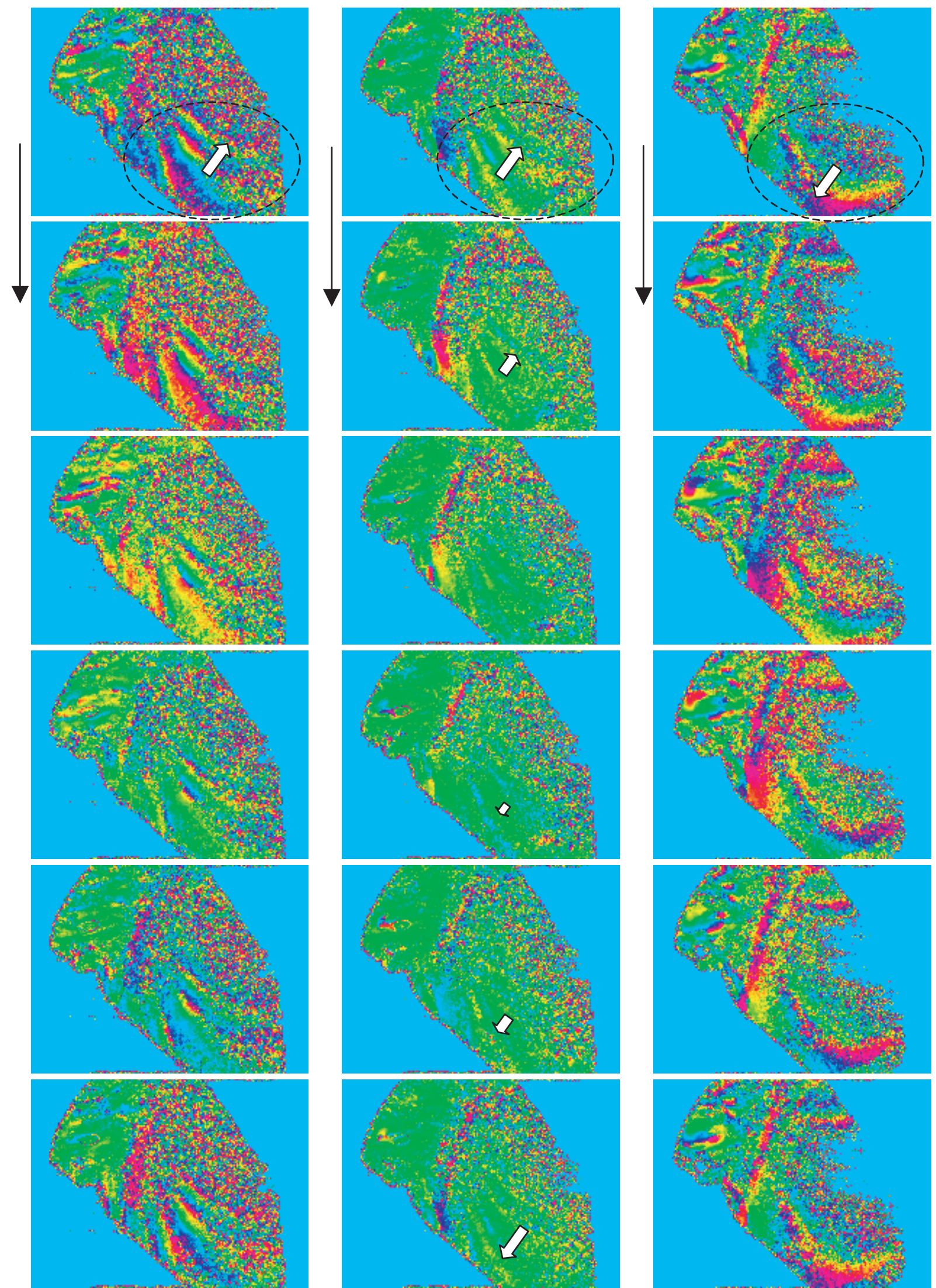

L-mode

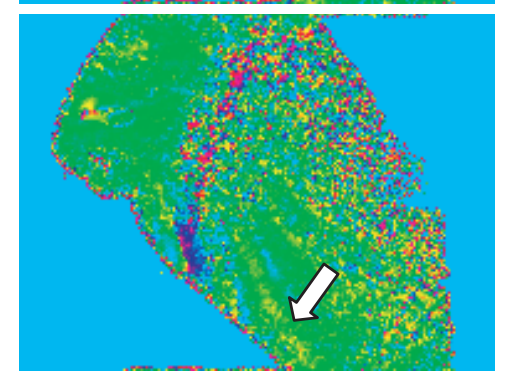

L-H transition
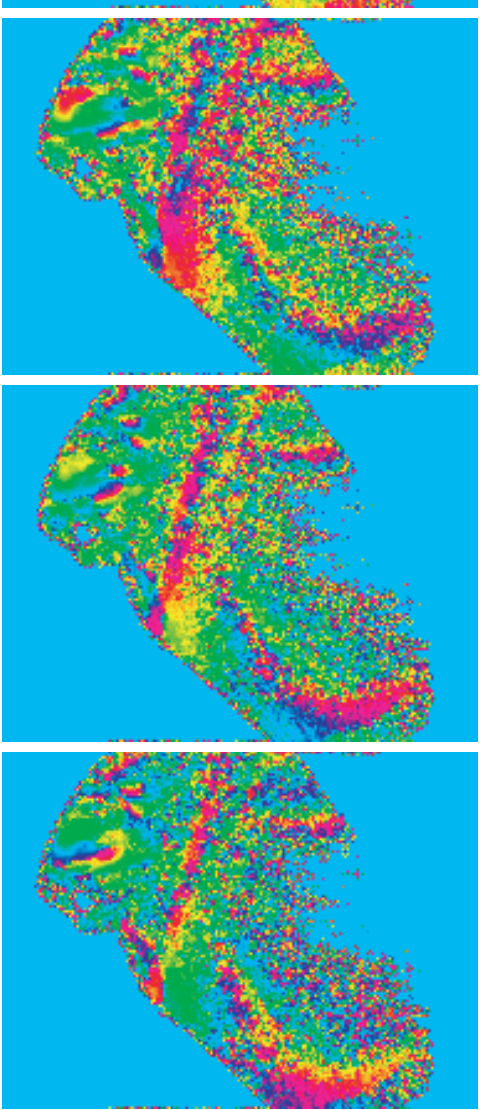

H-mode

Fig. 5 2D-Phase images during the L-mode, L-H transition, and H-mode Time progresses downward in each column, and time difference between each frame is $25 \mathrm{~s}$. Phase image in dashed circle region moves clockwise during the L-mode (see downward in left column). During the L-H transition, the motion of the phase image in dashed circle region become slow, and at the transition, the rotation of the phase image stops. After the transition, phase image begins to rotate but the direction is counterclockwise. 
32 frames for time-dependent FFT. The color corresponds to the phase range $-\pi$ to $\pi$. Regions with a particular color have the same phase. Therefore, these images show the propagation phenomena and/or wave at the selected frequency. The pictures in the left column show phase images during the L-mode, those in the center column shows phase images during the $\mathrm{L}-\mathrm{H}$ transition, and those in the right column show phase images during the H-mode. Time progresses downward in each column, and the time difference between each frame is $25 \mu \mathrm{s}$. The dashed ellipses show the top region of the vacuum vessel. As already mentioned, the emission from the peripheral plasma was easily measured in this region, and the filamentary structures in the $\mathrm{H}$-mode were seen to be somewhat wider than that in the L-mode. The rotation speed of $3000 \mathrm{~m} / \mathrm{s}$ was roughly estimated in the images during the $\mathrm{H}$-mode and it was about half during the L-mode. Therefore, according to this rough estimation from the images, $E_{\mathrm{r}}$ is $-2 \mathrm{kV} / \mathrm{m}$ during the $\mathrm{H}$ mode and $+1 \mathrm{kV} / \mathrm{m}$ during the L-mode. The magnetic field is about $1.5 \mathrm{~T}$. These results were consistent with Ref [7]. The period of the L-H transition was also deduced in the images. The number of camera frames in which plasma rotation stopped is only one at 40000 frames per second. This means that the transition period in Heliotron $\mathbf{J}$ plasma was below $50 \mu$ s (two frames), because the shutter was open in these experiments to observe light emission.

Figure 6 shows the ion saturation current of the movable probe of the same shot. The location of this probe is already shown in Fig. 1. The burst signal of the ion saturation current is maximum around $240 \mathrm{~ms}$ before the $\mathrm{H}$-mode began.

The power spectra of this ion saturation current are shown in Fig. 7. The fluctuations throughout the measurable frequency range were suppressed dramatically during the H-mode; however, the fluctuations were already suppressed from $240 \mathrm{~ms}$, especially in the low-frequency range.

$\mathrm{D} \alpha$ signal also decreased slightly from this time (see Fig. 3). During this period, the filamentary structures were seen to rotate mainly counterclockwise but sometime those structures stopped rotating and/or rotated clockwise for very short periods (a few frames). Therefore, in this period, it seems the L-mode plasma dither to shift to the $\mathrm{H}$ mode plasma. This phenomenon was named as "dithering phase" in Ref [7].

In the dithering phase, the diamagnetic signal increases with time (see Fig. 3) and the electron density also increased (not shown due to the signal jump after the $\mathrm{H}$ mode). The increments in both signals were about the same in an average of these H-mode shots. Therefore, plasma temperature seems to be constant, and the increment in plasma pressure is mainly due to the increment in the plasma density. The gas puff was carefully adjusted to obtain an H-mode plasma. However, as mentioned above, once the H-mode was obtained, the electron density increased rapidly and became uncontrollable. On the con-

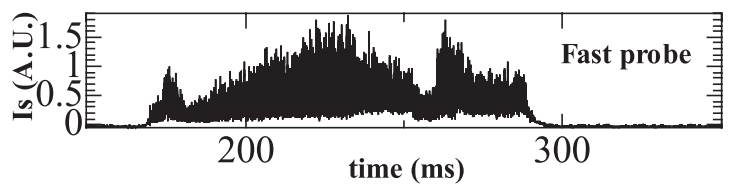

Fig. 6 Ion saturation current signal.

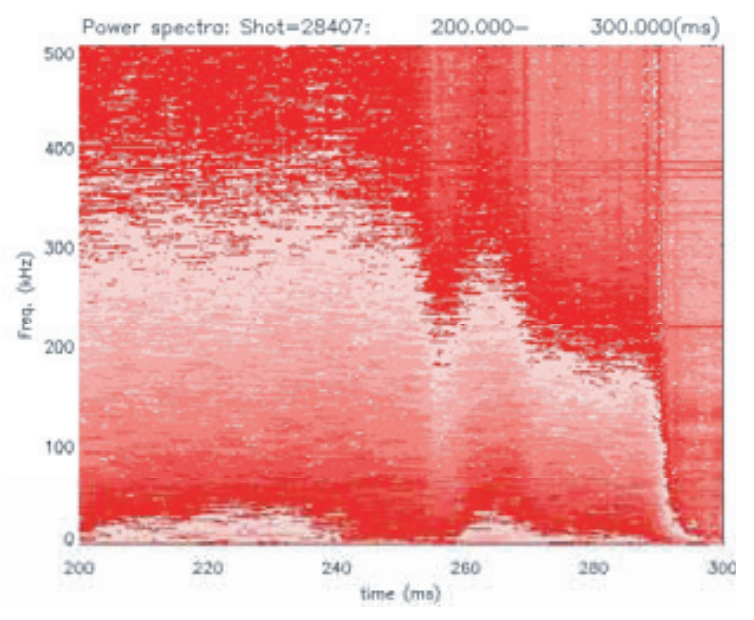

Fig. 7 Power spectra of the ion saturation current.

trary, in the dithering phase, the electron density seemed to be controllable, and this phase lasted more than the period of the H-mode plasmas. Therefore, to obtain high-pressure (or high- $\beta$ ) plasmas, this dithering phase might be preferable in Heliotron $\mathrm{J}$.

In these experiments, the $\mathrm{H}$-mode characteristics of Heliotron $\mathrm{J}$ were investigated and the motion of the filamentary structures during the dithering phase was identified. This dithering phase looks like the ELMy H-mode in tokamaks. We need to determine the difference between the dithering phase in Heliotron $J$ and the ELMy $\mathrm{H}$-mode in tokamaks. In the next experiment series, threedimensional turbulence measurement by more than two cameras will be demonstrated in Heliotron J. To compare these phenomena carefully, additional experiments will be planned very soon.

\section{Conclusion}

Using a fast camera, the peripheral turbulence of Heliotron J plasmas was measured successfully. In particular, the filamentary structure near the plasma edge was to seen to rotate. If this motion was poloidal rotation, the direction of this motion was changed during L-H transition, and this direction of rotation was consistent with the sign of $E_{\mathrm{r}}$ deduced from the past $\mathrm{H}$-mode experiments. Also, the transition period can be estimated by the fast camera images.

Use of the fast camera for three-dimensional plasma diagnostics will be started in Heliotron $\mathrm{J}$, and we believe the combination of the fast cameras and other diagnostics will be very helpful in obtaining important information on plasma behavior. 


\section{Acknowledgments}

This work is performed with the support and under the auspices of the Bi-directional Collaborative Research Program (NIFS04KUHL006).

[1] T. Obiki, T. Mizuuchi, K. Nagasaki et al., Nucl. Fusion 41, 883 (2001).

[2] N. Nishino, K. Takahashi, H. Kawazome et al., J. Nucl.
Mater. 337, 1073 (2005).

[3] N. Nishino, T. Mizuuchi, K.Kondo et al., J. Nucl. Mater. 363-365, 628 (2007).

[4] T. Mizuuchi et al., J. Nucl. Mater. 337-339, 332 (2005).

[5] T. Mizuuchi, W.L. Ang, Y. Nishioka et al., J. Nucl. Mater. 313-316, 947 (2003).

[6] N. Nishino, T. Mizuuchi et al., to be published in JFPR.

[7] F. Sano, T. Mizuuchi, K. Kondo et al., Nucl. Fusion 45, 1557 (2005). 